\title{
Gemeinden und Gemeindeverbände in der Dominikanischen Republik: Verfassung und Verfassungswirklichkeit in einem zentralisierten politischen System
}

\author{
Von Jürgen $H$. Wolff
}

\section{Einleitung}

Dezentralisierung der Verwaltung scheint in Entwicklungsländern und Entwicklungspolitik in den letzten Jahren in Mode gekommen zu sein, glaubt man politischen Kursbestimmungen und Absichtserklärungen. Autoritäre Regime pflegen den Versuch der Zentralisierung von Entscheidungen und daher in der Regel den Verfall lokaler Autonomie mit sich zu bringen1. Umgekehrt wäre dann zu erwarten, daß die gegenwärtige z.m. vordergründig der Demokratie günstige politische Entwicklung die Gemeindeautonomie begünstigte, überhaupt Dezentralisierung staatlicher Aufgabenerfüllung mit sich brächte. In der Tat sind hier weltweit beträchtliche Anstrengungen unternommen worden, übrigens mit gemischtem Erfolg2, da "Verwaltungsreform" ein hochkomplexer und hochpolitischer Vorgang ist, der nicht einfach auf den Faktor "Charakter des politischen Systems" reduziert werden kann. Auch die Entwicklungshilfe etwa der Bundesrepublik hat viel von ihrer früheren politischen Prüderie verloren: Die Förderung von Dezentralisierungsbemühungen im Rahmen der Verwaltungshilfe, ja selbst das Werben um solche Ideen im Rahmen des "Politikdialogs" bei Entwicklungshilfeverhandlungen sind häufiger als früher.

Ziel dieses Aufsatzes ist es, die gegenwärtige Situation der Gemeinden in der dominikanischen Republik außerhalb der Hauptstadt Sto. Domingo zu untersuchen. Auch in diesem Land ist der gemeindlichen "Autonomie" einige Rhetorik gewidmet worden, und die politische Entwicklung seit dem Sturz des Trujillo-Regimes oder mindestens dem Ende von Bürgerkrieg und US-amerikanischer Intervention 1965 ließe Dezentralisierung nicht als undenkbar erscheinen. Vorweggenommen sei das (auch schon im Untertitel angedeutete) Ergebnis: Bei aller Verbesserung etwa der finanziellen Lage der Gemeinden wird ihnen ein nennenswerter Entscheidungsspielraum nicht zugestanden; wohlwollender Paternalismus ist noch das Beste, was eine Kommune von der Zentralregierung zu erwarten hat. Die jahrhundertelange Tradition des Zentralismus ist inzwischen so sehr internalisiert worden, daß

1 Philip Mahwood, Hrsg., Local Govemment in the Third World. The Experience of Tropical Africa, Chichester et al., 1983.

2 Dennis A. Rondinelli, John R. Nellis u. Shabbir G. Cheema, Decentralization in Developing Countries, Washington 1984 (World Bank Staff Working Paper Nr. 581). 
ein anderer Stand der Dinge selbst für die betroffenen Bürgermeister im genauen Wortsinn undenkbar geworden ist.

Unser Thema soll folgendermaßen diskutiert werden: Der nächste Abschnitt sei der Situation der Gemeinden in der Dominikanischen Republik, der darauffolgende den Gemeindeverbänden, insbesondere der Liga Municipal Dominicana gewidmet. Schließlich wird der Versuch gemacht, die Rückbeziehung zur Zentralisierung und der politischen Lage zu erläutern.

Soweit keine anderen Quellen angegeben werden, stütze ich mich auf Interviews im Rahmen einer Prüfmission für die GTZ im Herbst 1987.

\section{Die Situation der Gemeinden in der Dominikanischen Republik}

\section{Zur Entwicklung der Siedlungsstruktur}

Die spanische Kolonie und die unabhängige Republik sind über Jahrhunderte hinweg durch eine Bevölkerungsverteilung charakterisiert gewesen, die vom üblichen Schema einer mehr oder weniger großen Konzentration auf einen Kernraum (die Hauptstadt) abweicht: Sto. Domingo, die koloniale wie moderne Hauptstadt, liegt isoliert an der Südküste, und die stärkste Bevölkerungskonzentration findet sich in der agrarisch reichen Landschaft des Cibao, die von Sto. Domingo aus auf dem Landwege nicht - oder allenfalls über schwierige Maultierpfade - erreicht werden konnte.

Das Fehlen von leistungsfähigen Straßen und der geringe Bevölkerungsdruck auf dem Lande läßt dieses Grundmuster bis in die 20er Jahre unseres Jahrhunderts unverändert. Sto. Domingo als größte Stadt weist noch 1920 eine Bevölkerungszahl von nur 31.000 auf; nur 6 weitere Städte zählen mehr als 5.000 Einwohner. Ein Anteil der städtischen Bevölkerung in diesen sieben Städten an der Gesamtbevölkerung des Landes liegt unter $10 \% 3$ von 895.000 .

Seither verläuft die Entwicklung in den für zahlreiche Länder der Dritten Welt typischen Bahnen; die enorme absolute Zunahme der Bevölkerung (1981 5,648 Millionen; 1985 nach Fortschreibung 6,243 Millionen, s. hierzu Statistisches Bundesamt 1986) geht mit einer immer stärkeren Konzentration auf die Städte, voran Sto. Domingo, einher. 1985 wohnten bereits fast $56 \%$ der Bevölkerung in Städten, nur noch $44 \%$ auf dem Lande. Sto. Domingo wuchs von 370.000 Einwohnem 1960 auf 1,31 Millionen 1981; neuere Schätzungen bzw.

3 Oficina Nacional de Planección (ONAPLAN) El Proceso de Urbanización en le República Dominicana, Nov. 1983. 
Hochrechnungen geben der Hauptstadt 1987 1,82 Millionen Einwohner, was etwa 28\% der Gesamtbevölkerung des Landes entspricht.

Stagniert die Zahl der Einwohner auf dem Lande seit etwa 1975, so steigt doch die Einwohnerzahl der mittleren und kleineren Städte seit 1960 mit Raten an, die weit über der Zunahme der Gesamtbevölkerung liegen. Allerdings ist - wie für die Bevölkerung insgesamt - in den letzten Jahren ein deutlicher Rückgang der Zuwachsraten zu verzeichnen.

Wie auch in zahlreichen Studien für andere Länder Lateinamerikas belegt, liegen die Hauptursachen für die Wanderungsströme in unterschiedlichen Lebensbedingungen auf dem Lande und in der Stadt. Zwischen Zuwanderern und auf dem Lande Zurückbleibenden zeigen sich charakteristische Unterschiede, die auf Dauer die Attraktivität des Landes noch mehr mindern müssen.

Als Ergebnis der demographischen Entwicklung und der Wanderungsbewegung weist die Dominikanische Republik heute mehrere deutlich abgegrenzte städtische Subsysteme auf: Das Subsystem "Cibao" (Cibaotal); das Subsystem "Südküste" (zw. Barahona und La Romana) und das Subsystem Zentralkorridor Sto. Domingo - Santiago.

Daneben ist im Westen S. Juan mit seiner Einflußzone zu nennen. Nach ihrer Größenordnung können fünf Stadtklassen unterschieden werden (Sto. Domingo; Santiago; 20-100.000 Einwohner, 17 Fälle; 10-20.000 Einwohner, 11 Fälle; weniger als 10.000 Einwohner, 96 Fälle).

\section{Die Ausstattung der dominikanischen Gemeinden}

Die angedeutete Bevölkerungsentwicklung hat ihrerseits eine überproportionale Konzentration der Bauwirtschaft und der öffentlichen Investitionen auf die größeren Zentren mit sich gebracht. In zirkulärer Verursachung hat die relativ bessere Ausstattung der größeren Gemeinden deren Attraktivität als Wanderziel erhöht. Das Ergebnis ist eine noch größere Diskrepanz zwischen der physischen Infrastruktur der größten und großen Städte und der jenigen der Klein- und Mittelstädte.

Eine Untersuchung des staatlichen Planungsamtes von 15 Mittelstädten aus dem Jahre 19834 , in dem für die Bereiche Trinkwasserversorgung, Abwasserbeseitigung, Versorgung mit elektrischem Strom, Straßenbeleuchtung und Pflasterung der Ortsstraßen nach

4 Liga Municipal Dominicana, (LMD) Recopilación de Disposiciones Legales relacionadas con la Vida Municipal, 2. A., Sto. Dorningo, 1983. 
bestimmten Kriterien Noten vergeben wurden (zur Methodologie vgl. die Quelle), weist aus:

Von 75 vergebenen Urteilen wurde erteilt die Note:

gut (bueno)

(mit Ausnahme alle im Bereich der Wasserversorung);

befriedigend

ausreichend (regular)

schlecht

nicht vorhanden
$6 \mathrm{mal}(8 \%)$;

$21 \mathrm{mal}(28 \%)$;

$21 \mathrm{mal} \mathrm{(28 \% );}$

$23 \mathrm{mal}(31 \%)$;

$4 \mathrm{mal}(5 \%)$.

Ohne daß parallele Untersuchungen in den kleineren Gemeinden bekannt geworden wären (vgl. aber detaillierte Regionalplanungsstudien der ONAPLAN von Ende der 70er und Anfang der 80er Jahre), kann davon ausgegangen werden, daß mit abnehmender Gemeindegröße die physische Ausstattung - zu denken wäre auch an medizinische Versorgungseinrichtungen, Schulen, Märkte usw. - immer lückenhafter wird.

\section{Zur administrativ-politischen Infrastruktur der dominikanischen Gemeinden:}

Im Laufe der Jahrzehnte ist zu den Gemeinden in der Dominikanischen Republik eine umfangreiche Gesetzgebung entstanden. Die vom Gemeindeverband Liga Municipal (zu dieser s. unten) besorgte Zusammenstellung 5 umfaßt nicht weniger als 743 Seiten!

Einschlägig sind zunächst verschiedene Artikel der Verfassung. Art. 82 legt den Grundsatz der Wiederholung der staatlichen Organisationsstruktur auf der Ebene der Munizipien fest. Ein Bürgermeister (Síndico) wird alle vier Jahre direkt durch die Wähler gewählt, desgleichen ein Stadtrat (Sala Capitular). Beide Gewalten (die lokale Exekutive und die lokale Legislative) sind also, wie im Präsidialsystem der Gesamtrepublik, voneinander unabhängig und stützen sich jeweils auf eigene Legitimitätsquellen, direkte Wahl durch das Volk.

Art. 83 bestimmt die Unabhängigkeit der Gemeinden bei der Ausübung ihrer Aufgaben, stellt diese aber unter Gesetzesvorbehalt (ebenso Art 85 für die gemeindlichen Finanzen). Einer immer weiterreichenden Zentralisierung von Verwaltung und Politik gegenüber haben die Gemeinden also nicht einmal die Verfassung auf ihrer Seite!

5 Christoph Sigrist, Inforne provisional sobre el sistema e finanzas intergubemamentales y la incidencia de la ley N.140 en la República Dominicana y en el Distrito Nacional, hekt., o.d. - ca. 1984, S.21. Statistisches Bundesamt, Länderbericht Dominikanische Republik, Stuttgart und Mainz, 1986. 
"Gemeinden" sollten nicht ohne weiteres mit dem deutschen Begriff gleichgesetzt werden. Eine dominikanische Gemeinde umfaßt in der Regel ein größeres Territorium mit einem Hauptort und mehreren Siedlungskemen (Art. 54 Ley 3455), für die u.U. Dorfbürgermeister (Alcaldes Pedáneos) zuständig sind. Der "Districto Municipal" hingegen wiederholt als Teil einer Gemeinde dessen Organisationsstruktur, bleibt aber unter der Überwachung der Gesamtgemeinde (Kap. IV Ley 3455).

Grundlegend für die Organisation der Gemeinde ist Gesetz 3455 aus dem Jahre 1952 (Neufassung in Vorbereitung, s. unten). Es seien im folgenden nur einige im vorliegenden Zusammenhang wesentliche Grundsätze wiedergegeben (allein das zit. Gesetz umfaßt 222 Artikel auf 77 Seiten!): Jede Gemeinde stellt eine juristische Person mit eigenem Einkommen und Vermögen dar (Art. 1); der Stadtrat wählt jährlich seinen Präsidenten (Art. 16); das Amt des Stadtrates ist ehrenamtlich (Art. 13), nicht hingegen dasjenige des Bürgermeisters.

Dem Stadtrat kommt eine eindrucksvolle Reihe von Aufgaben zu (Art. 31), die sich bei näherem Zusehen indes auf wenige Bereiche von Bedeutung reduzieren:

- Aufgaben der Stadtplanung und Baü̈berwachung;

- Versorgung mit Trinkwasser, Bau von Abwasserleitungen;

- Aufgaben einer Gesundheitspolizei und der örtlichen Verkehrspolizei;

- einige wenige Aufgaben im Bereich der öffentlichen Ordnung;

- Aufgaben im Bereich des Haushaltes und der Finanzverwaltung;

- Emennung der Gemeindebediensteten.

Der Síndico ist Leiter der Gemeindeverwaltung und der Finanzverwaltung (Art. 34ff.); überraschenderweise hat er die Aufgabe der Kontrolle von Maßen und Gewichten (Ziff. 18 idem). Ohne Zustimmung des Stadtrates und des Innenministers darf er übrigens die Gemeinde nicht verlassen (Art. 36).

Mit Finanzangelegenheiten ist der Kämmerer (tesorero) beauftragt (Art. 38ff.), neben dem Sekretär des Gemeinderates (Art. 42ff.) das einzige Amt außer dem Bürgermeister, über das jede Gemeinde verfügen muß. Inspektoren (Art. 64ff.), eine Art Gemeindepolizei, sind fakultativ.

Die verbleibenden Vorschriften des zitierten Gesetzes regeln bis ins Detail das Finanzgebaren und die Haushaltsdurchführung der Gemeinden. Eine Wiedergabe der komplizierten Vorschriften dürfte sich erübrigen; hier gilt, daß ihre Bedeutung im umgekehrten Verhältnis zu den verwalteten Werten - selbst unter Berücksichtigung der relativen Armut des Landes - steht. 
Das Entstehungsjahr des Gesetzes erklärt, warum bestimmte, im Detail zu besprechende Kontrollrechte der Liga Municipal gegenüber den Gemeinden nicht vorkommen. So hat der tesorero (gem. Ziff. 7 Art. 38) zwar die Rechnungsprüfung durch Controlaría General und Cámara de Cuentas, nicht aber diejenige durch die Liga zu ermöglichen: Die entsprechenden Vorschriften sind erst lange nach der Verabschiedung des Gemeinde-Grundgesetzes erlassen worden. Der vorliegende Gesetzesentwurf zur Aktualisierung des Gemeinderechts inkorporiert die zwischenzeitliche Gesetzgebung, wobei schon die kaum überschaubare Regelungsvielfalt die Gemeindeautonomie durch die Kontrollrechte der Liga Municipal stark eingeschränkt erscheinen läßt.

\section{Zur finanziellen und personellen Ausstattung der Gemeinden und zu Grundlinien ihrer Organisation}

\section{a. Die Gemeindehaushalte}

Bei den kommunalen Einnahmen zu unterscheiden sind grundsätzlich eigene Einnahmequellen der Gemeinden und Transferzahlungen der Regierung.

Die von der Liga Municipal Dominicana verwendete Einnahmen-Klassifikation unterscheidet eine große Zahl von - in der Mehrzahl der Gemeinden bedeutungslosen - Einnahmequellen, die in folgende große Gruppen zusammengefaßt sind:

- Steuern (impuestos), meist (im deutsche Sinne) Verkehrssteuern;

- Gebühren (tasas), etwa für Baugenehmigungen;

- Verschiedene Einnahmen (rentas diversas), z.B. für die Verpachtung von Grundstükken, die Lieferung von Wasser und Strom, Vermietung von Baumaschinen (maquinaria pesada);

- Einkünfte aus eigenen Einrichtungen (Proventos y Establecimientos Productivos);

- Mahn- und Überziehungsgebühren (recargos);

- Sonstige (otros ingresos especiales y diversos), z.B. Verkauf von Grundstücken, Bürgschaft für die Bezahlung von elektrischem Strom.

Hinzukommen verschiedene Kategorien offener Einnahmepositionen (noch nicht realisierte Ansprüche der Gemeinden), eine reine Rechnungsabgrenzung, aber keine eigene Einnahmequelle.

Zwei Dinge fallen ins Auge: Die Einnahmesystematik entspricht nicht den akzeptierten Kriterien der Finanzwissenschaft. Die Abgrenzung zwischen Steuern, Gebühren, Beiträgen erscheint unklar; unter einer Rubrik werden verschiedene Einnahmearten (im abgabensystematischen Sinne) zusammengefaßt. 
Zum anderen hat die Klassifikation wenig bis nichts mit der Klassifikation im Gemeindegrundgesetz_ (3455, Art. 121ff.) zu tun. Stellt etwa Art. 123 idem die Einführung und Veränderung von "arbitrios" unter den Genehmigungsvorbehalt des Innenministers und nimmt Art. 124 wiederum bestimmte Arten vnn "arbitrios" davon aus, dann bleibt offen, welche der oben angeführten Einnahmearten wohl mit diesem Terminus gemeint sein mögen. Die Praxis indes hat die Kontrollrechte der Liga und des Innenministers, die reinen Gebühren (z.B. für Müllabfuhr) ausgenommen, ordentlich verstärkt.

Eine Durchsicht und Aktualisierung des gemeindlichen Abgabenrechtes erscheint als eine dringliche Aufgabe. Insbesondere eine Aktualisierung des Gebührenrechtes für die hoch defizitären Leistungen der Gemeinden dürfte sich anbieten.

Die wichtigste Einnahmequelle der Gemeinden sind hingegen Transferzahlungen. Hatte bereits Gesetz 624 (1973) für jede Gemeinde des Landes einen monatlich zu zahlenden "subsidio ordinario" festgelegt (als absolute Werte selbstverständlich nach einigen Jahren durch die Inflation aufgezehrt), so wird durch Gesetz 140 (1983) mit Wirkung vom Jahre 1984 grundsätzlich den Gemeinden ein bestimmter Anteil an den Staatseinnahmen zugestanden: Der Staat zahlt ihnen 20\% des Aufkommens der "Rentas internas", im wesentlichen das inländische Steueraufkommen ohne die besonders ertragreichen Steuern auf Einkommen und Umsatz. Überschlägig gerechnet, machten diese 1986 nicht ganz 20\% der Staatseinnahmen aus, so daß die Gemeinden ca. $4 \%$ des Staatshaushaltes als Transferzahlungen erhalten.

Hinzugefügt sei, daß die Liga Municipal einen bestimmten Prozentsatz hiervon für die eigenen Verwaltungsaufwendungen erhält; eine zusätzliche Klausel soll sicherstellen, daß gegenüber der vorherigen Transferleistung in keinem Falle eine Verschlechterung eintritt.

Die Auszahlung ist von Gesetzes wegen (Art. 7) zehn Tage nach Eingang durch die Liga Municipal, d.h., spätestens am 20. eines jeden Monats vorzunehmen (Strafvorschriften bei Zuwiderhandlung in Art. 11). Dies geschieht indessen nicht in allen Fällen; vielmehr wird die Verzögerung dieser den Gemeinden zustehenden Transferzahlung genutzt, um die Einhaltung haushaltsrechtlicher Bestimmungen oder die Vermeidung übergroßer Verschuldung der Gemeinden zu erzwingen.

Gesetz 140 legt (in Art. 9) fest, daß maximal 50\% der Gemeindehaushalte für Gehälter ausgegeben werden dürfen bzw. mindestens $25 \%$ für Dienstleistungen und Unterhalt und $25 \%$ für Investitionen ausgegeben werden müssen. Die Einhaltung dieser Vorschriften stellt die Haushaltskontrolle der Liga sicher.

Den Gemeinden ist auf Verlangen durch das Finanzministerium die notwendige Information über die Durchführung des Gesetzes zu geben (Art. 10). Soweit festgestellt werden 
konnte, werden solche Informationen jedoch nicht erteilt, die Gemeinden werden monatlich von der Liga mit einem Scheck bedacht, ohne daß die Berechnung des Betrages von ihnen nachvollzogen werden könnte.

Gesetz 140 hat - neben der besseren und vor allem neben der dynamisierten Finanzausstattung der Gemeinden - bemerkenswerte Folgen: Zum einen wird, da der überwältigende Teil der Einnahmen aus Transferleistungen stammt, tendenziell der Anreiz zur Erschließung eigener Einnahmequellen vermindert. Es unterliegt keinem Zweifel, daß hier beträchtliche Reserven mobilisiert werden könnten. Zum anderen wird die Berechnung der Transfers mechanisch nach der Einwohnerzahl der Gemeinden vorgenommen. Damit ist automatisch ein erhebliches Element eines horizontalen Finanzausgleiches gegeben.

Nehmen wir zur Veranschaulichung etwa an, zwei Gemeinden gleicher Einwohnerzahl hätten eigene Einnahmen, die sich wie 1:2 verhalten, und nehmen wir weiter an, beide erhielten eine gleichhohe Transferleistung, die viermal so hoch ist wie die Einnahmen der ersten Gemeinde: Das simple Ergebnis ist, daß der Unterschied der verfügbaren Finanzmassen vom Doppelten auf 1/5 reduziert wird: Die reiche Gemeinde verfügt nur noch über $20 \%$ mehr Einnahmen als die ärmere. In einem derart zentralisierten Staat wie der Dominikanischen Republik ist ein solches Ergebnis von nicht zu unterschätzender Bedeutung.

Eine Analyse ausgewählter Gemeindehaushalte bestätigt die Richtigkeit der angeführten Überlegung:

Die verfügbare Finanzmasse der Gemeinden pro Kopf differiert nur geringfügig. Mindestens das Problem des horizontalen Finanzausgleichs dürfte für die Dominikanische Republik als gelöst anzusehen sein. Damit ist freilich der Nachteil verbunden, daß die Gemeinden in ihrer Finanzausstattung weitestgehend von staatlichen Zuwendungen abhängen; über deren Veränderung - die freilich dem Gesetz nach nicht selektiv für Einzelgemeinden vorgenommen werden darf - ist der Aktionsspielraum der Gemeinden zu erweitern oder żu begrenzen.

Eine makrofiskalische Analyse bestätigt, daß die Streuung der verfügbaren Einnahmen der Gemeinden pro Einwohner nur geringfügig ist. Um eine Vorstellung von den geringen absoluten Werten zu vermitteln, seien die entsprechenden Zahlen hier wiedergegeben:

$$
\begin{aligned}
\text { (Kurs im September 1987: } 1 \text { RD } \$=0,29 \text { US } \$ \\
1 \text { US } \$=3,45 \text { RD\$) }
\end{aligned}
$$

Gesamthaushalt aller Gemeinden ohne

Santiago und Sto. Domingo (1986)

Bevölkerung ohne Santiago und Sto. Domingo 
Transferzahlungen des Staates nach Gesetz 140

(e. Sto. Domingo u. Santiago)

RD\$

67.283 .213

d.s. pro Einwohner (e. Sto. Domingo u. Santiago) RD\$

10,26

Bei diesen Werten ist noch zu beachten, daß die Transferzahlungen nach Gesetz 140 teilweise auch zur Finanzierung des Gemeindetages Liga Municipal verwendet werden.

Die pro Kopf gleichen Transferleistungen des Staates ab 1984 stehen in deutlichem Gegensatz zur vorherigen Situation, die die Ballungszentren bevorzugte. Es ist zu erwrten, daß im Laufe der Jahre eine Angleichung der infrastrukturellen Ausstattung der Gemeinden jedenfalls in den Bereichen eintreten wird, die von den Lokalverwaltungen bestimrnt werden.

Auch wenn es kaum mehr eine Benachteiligung wirtschaftsschwacher Gemeinden in der Finanzausstattung gibt, sind die Problembereiche "ungenügende eigene Einnahmen" und "geringe absolute Höhe der verfügbaren Finanzmittel" ungelöst. Für Gemeinden typische Einnahmearten, voran die Grundsteuer, werden nicht oder nur in ganz ungenügender Weise erschlossen (dem Kongress liegt allerdings ein entsprechender Gesetzentwurf vor). Gebühren sind viel zu gering.

Ein Gesetzentwurf, der eine Erhöhung des an die Gemeinden zu zahlenden Anteils der "Rentas Internas" von $20 \%$ auf $25 \%$ vorsieht, liegt dem Kongreß vor.

Die Folge ist, daß zahlreiche der oben dargelegten Gemeindeaufgaben nicht oder nur in unzureichender Weise erfüllt werden könnten. Eine Stadtplanung, die den Namen verdiente, gibt es nur in den größeren Städten Santiago und Sto. Domingo, nicht aber in den kleineren Städten. Dies steht im Widerspruch zum Gesetz 6232 (1963), das für Städte mit mehr als 55.000 Einwohnern eine detaillierte Stadtplanung (planos pilotos, planos reguladores usw.) und die entsprechende administrative Infrastruktur vorgeschrieben hat.

Die Trinkwasserversorgung, soweit vorhanden, wird wesentlich durch eine parastaatliche Einrichtung (Instituto Nacional de Aguas Potables y Alcantarillados, INAPA) wahrgenommen. Die Müllabfuhr funktioniert in den Siedlungskernen mäßig bis schlecht, auf dem Lande in der Regel überhaupt nicht. Das Fehlen von Abwasserleitungen in zahlreichen Ortschaften stellt ein Risiko für die menschliche Gesundheit und für die Umwelt dar.

Alle diese Probleme sind allerdings im Verhältnis zu manch anderen lateinamerikanischen Ländern zu relativieren: Das hohe Bruttosozialprodukt von US\$ 1380 pro Kopf 1983 zeigt sich auch im Bereich der physischen Infrastruktur der Gemeinden, die weit über der vergleichbarer Munizipien etwa in El Salvador, Paraguay oder Guatemala liegt. 
b. Ausgabenstruktur

Auf die Vorschriften des Gesetzes 140, das Obergrenzen für Personalausgaben und Mindestausgaben für gemeindliche Investitionen vorsieht, wurde bereits hingewiesen. Der Einhaltung gerade dieser Normen widmet die Kontrollabteilung des Gemeindetages besondere Aufmerksamkeit. Wenigstens im Budget dürften Abweichungen nicht möglich sein.

Die der Haushaltskontrolle der Liga zugrundegelegte Haushaltsklassifizierung erscheint im Detail kompliziert: In einem ersten Schritt wird "Funktion und Organisationseinheit" mit "Ausgabeart" (objetivo de gasto) gekreuzt. Die Klassifikationserläuterung legt allerdings klar, daß "Funktion" und "Organisationseinheit" gleichgesetzt werden.

Vier Ausgabengruppen (mit zahlreichen Untergruppen) werden unterschieden:

- Verwaltung;

- gemeindliche Investitionen (obras públicas municipales);

- verschiedene gemeindliche Dienstleistungen (servicios municipales diversos);

- unklassifizierbare.

Als Ausgabenarten werden aufgeführt (wiederum mit zahlreichen Unterpunkten):

- Personalkosten

- sonstige laufende Ausgaben

- Dienste und vertragliche Leistungen (obras contractadas)

- Erwerb von Aktiva

- unklassifizierbare.

Sonderaufstellungen zeigen bis ins Detail die Gehälter, Renten, Subventionen, Erwerb von Aktiva und Sonderfonds.

Die aufgeführten Klassifikationen sind von kleinen Gemeinden kaum zu handhaben. Die Liga nimmt infolgedessen auch Beratungsaufgaben wahr. Der außenstehende Beobachter fragt sich allerdings, ob ein Beratungsbedarf nicht erst durch die komplizierten Vorschriften, für die u.a. die Liga selbst verantwortlich ist, hervorgerufen wurde.

Ganz grob kann davon ausgegangen werden, daß von den klassifizierbaren Ausgaben (die Restkategorie "Unklassifizierbar" kann beträchtliche Anteile ausmachen!), ein starkes Drittel für Dienstleistungen, ein Viertel für Investitionen und der Rest für Verwaltung ausgegeben wird.

Die Haushaltsklassifikationssysteme der verschiedenen mit Haushaltsüberwachung und kontrolle befaßten Stellen unterscheiden sich beträchtlich. Die Liga Municipal verwendet 
zur Uberwachung der Vorschriften des Gesetzes 140 (Ausgabenhöchstsätze, s.o.) ein System, das von ihrem eigenen abweicht; die beiden Rechnungshöfe, die wir oben erwähnt haben, verwenden wiederum eine jeweils verschiedene Systematik. Man kann sich unschwer ausmalen, welche Arbeitsbelastung die komplizierten Systeme für die kleinen Gemeinden mit sich bringen.

Auf ein in Entwicklungsländern geläufiges Phänomen, das auch in ausgeprägter Form in der Dominikanischen Republik beobachtet werden kann, sei lediglich verwiesen:

Der Haushalt und seine Durchführung unterscheiden sich in weit größerem Maße, als dies in Industrieländern vorkommt. Technische wie politische Ursachen wirken hierbei zusammen. Eine vertiefte Analyse der Gemeindefinanzen wird daher, soweit erhältlich, auf den Haushaltsrechnungen, nicht hingegen den Budgets beruhen müssen.

c. Personalausstattung der Gemeinden und ihre Organisation

Zu trennen sind grundsätzlich:

- Politische Ehrenämter wie diejenigen der Stadträte (Art. 13 Ley 3455). Ihre Zahl wechselt bei einem Minimum von fünf mit der Größe des Ayuntamiento (ein Stadtrat pro 14.500 Einwohner, Art. 5 Ley 5379, 1960). Inkompatibilitätskriterien sollen Interessenkonflikte verhindern; grob gesprochen kann Stadtrat nur werden (Art. 8 idem), wer nicht in einem Arbeitsverhältnis steht, und auch nur, wer keine Schulden gegenüber der Gemeinde hat.

- Politische Wahlämter, voran das des Síndico und das des Leiters (presidente) des Stadtrates;

- Festangestellte Bedienstete (personal nombrado), der Kern der täglichen Verwaltung;

- Tagelöhner (jomales), z.B. Straßenkehrer;

- Die "Alcaldes pedáneos" (Dorfbürgermeister), die je nach Möglichkeiten und Zweckmäßigkeiten nach Auffassung des Municipio ein mehr oder weniger großes Entgelt erhalten können (Art. 63 Ley 3455) bzw. nach der neueren Gesetzgebung (Gesetz 5972, 1962) dann erhalten, wenn dies im Haushaltsgesetz festgelegt ist.

Eine im Detail hier nicht wiederzugebende Analyse der Personalstruktur ausgewählter Gemeinden läßt folgende Schlußfolgerungen zu: 
1. Wie nicht anders zu erwarten, steigen Anzahl und Anteil der qualifizierten (und daher besser bezahlten) Mitarbeiter mit der Gemeindegröße. Logischerweise steigt damit die Durchschnittsentlohnung. Die Gesamtbelastung für den Haushalt mag dennoch geringer sein.

2. Erstaunlicherweise ist festzustellen, daß die Spannweite der Entlohnung von Mitarbeitern mit annähernd gleichen Funktionen von einer Gemeinde zur nächsten gering ist. Offensichtlich kommt hierin eine egalisierende Wirkung der verschiedenen Gemeindeüberwachungsorgane (vor allem in finanziellen Angelegenheiten) zum Ausdruck.

3. Die Gemeinden nehmen - mit oder ohne (bzw. gegen) gesetzliche Vorschriften - bei den von ihnen angebotenen Dienstleistungen ein immer noch großes $\mathrm{Maß}$ an Gestaltungsfreiheit in Anspruch. Leistet sich etwa eine Gemeinde eine Berufsfeuerwehr und strebt eine (allerdings von der Liga Municipal beanstandete) Musikschule an, so betont die andere planerische Aspekte und unterhält ein ausgebautes technisches Büro.

4. Große Munizipien sind im einzelnen besser ausgestattet, nehmen aber nicht unbedingt zusätzliche Aufgaben wahr.

5. Die Explosion von Personalkosten wird durch die zitierten Vorschriften verhindert. Nach den Berechnungen der Liga Municipal liegen sie für unsere Beispielsgemeinden zwischen 39 und $49 \%$.

Zwei Einschränkungen sind allerdings zu machen: Zum einen ist im Verhältnis zu anderen lateinamerikanischen Ländern, z.B. Paraguay, die Obergrenze von 50\% hoch angesetzt. Zum anderen sind die Gemeinden selbstverständlich verpflichtet, die gesetzlichen Mindestlöhne zu zahlen. Da zahlreiche Bedienstete kaum mehr als den Mindestlohn erhalten, schlägt jede Erhöhung voll auf die Ausgaben der Gemeinden durch.

Steigen die erwarteten Einnahmen (insbesondere die Transferzahlungen des Staates) nicht im gleichen Umfange und beträgt der Anteil der Personalausgaben am Gesamthaushalt bereits fast die Hälfte, dann bleibt den Gemeinden nur die Entlassung von Mitarbeitem als Ausweg. Für die anderen Gemeinden kommt es zu einem Absinken des Anteils von Anschaffungen und Investitionen.

6. Ein Laufbahn-Beamtentum auf Gemeindeebene gibt es nicht (dem Vemehmen nach liegt z.Z. dem Kongreß ein entsprechender Gesetzentwurf vor); der Stadtrat ist berechtigt, die Bediensteten der Gemeinden zu "emennen" (Ziffer 63 Art. 31 Ley 3455). Uber die Entlassung schweigt das Gesetz sich aus, gemeint dürfte indes sein, daß das Emennungsrecht das Entlassungsrecht umfaßt. 
In der Praxis wird mit dem Wechsel des Bürgermeisters und der Neuwahl des Stadtrates - vor allem wenn die politischen Mehrheitsverhältnisse sich verändem - ein weitreichender Personalaustausch vorgenommen. 1986 trat dies in zahlreichen Gemeinden ein; eine ordnungsgemäße Übergabe der Geschäfte erfolgte häufig nicht; selbst Akten waren unauffindbar, gelegentlich verschwanden auch Teile der Büroausstattung.

7. Zur Qualität der Ausbildung von Gemeindebediensteten sind generalisierende Aussagen schwierig, da es an entsprechenden Untersuchungen fehlt. Immerhin fällt auf, daß Mindestanforderungen an die Qualifikation nur für den Bürgermeister und die Stadträte bestehen (Art. 7ff., Ley 3455). Ein analphabetischer Kämmerer, wie er angeblich in einer Gemeinde arbeitet (und eine hervorragende Begründung für Kontrollmaßnahmen durch die Liga abgibt), scheint dem Gesetz nach zulässig zu sein. Die Communis opino unter Fachleuten ist jedenfalls, daß die Qualität der Gemeindebediensteten mäßig sei.

8. In unseren "Mustergemeinden" ergibt sich 1 Bediensteter auf 1283 Einwohner, ein für Lateinamerika durchschnittlicher Wert. Eine überschlägige Hochrechnung für das ganze Land (Statistiken waren nicht verfügbar) ergibt eine Gesamtzahl von ca. 3440 Gemeindebediensteten (ohne Santiago und Sto. Domingo).

\section{d. Aus- und Fortbildung durch die Liga}

Eine Abteilung für Fortbildung der LMD wurde Anfang 1987 ersetzt durch eine Verwaltungsschule (Escuela de Capacitación Municipal) und ein Institut für Verwaltungsstudien. Eine gesonderte Abteilung für Fortbildung besteht seither nicht mehr. Aus dem Tätigkeitsbericht geht hervor, daß 1986 durch die alte Abteilung eine dreitägige Vortrags- und Diskussionsrunde über Gemeindeverwaltung mit 74 Teilnehmem veranstaltet wurde. Außer der zweiten Aktivität, der Neustrukturierung, wird nichts weiter berichtet. Die Schule gehört dem Sekundarschulbereich an. Ihrer Gründung durch die Liga sind Untersuchungen des Ausbildungsbedarfs nach Umfang und Spezialisierungsrichtung nicht zugrundegelegt worden. Nimmt man für die Schule bei einer Jahrgangsstärke von 90 Schülem einen vorzeitigen Abgang (drop-out) von 1/3 an, dann würden pro Jahr 60 Schüler mit "Verwaltungsabitur" auf den Markt drängen.

Ein Blick auf die Personalstruktur der Gemeinden im Detail zeigt, daß für diese Aspiranten z.Z. kein Raum ist: Für manuelle Hilfsdienste sind sie überqualifiziert; für technische Aufgaben nicht ausgebildet; für Spezialaufgaben etwa im Bereich der Finanzverwaltung nicht zu gebrauchen, weil bei derart "politischen" Aufgaben Achtzehnjährige nicht akzeptiert werden. Selbst wenn man annähme, sie könnten entsprechende Fertigkeiten in einigen Monaten in Schnellkursen erlemen (warum aber dann ein spezielles Verwaltungsabitur?), 
werden auf keinen Fall Jahr für Jahr 60 solche Positionen frei. Die Produktion "sub-akademischer" Arbeitsloser ist programmiert!

\section{Gemeindeverbände, insbesondere die Liga Municipal Dominicana}

Für verschiedene Regionen des Landes (Norden; Nordwesten; Süden) existieren Regionalentwicklungsgesellschaften, an deren Organen die dominikanischen Gemeinden beteiligt sind, ohne bestimmenden Einfluß auszuüben. Wie bei der zentralstaatlichen Oficina de Desarrollo de la Comunidad wird die Geschäftspolitik der noch jungen Gesellschaften von der Regierung bestimmt. Auch ihr Etat stammt aus Mitteln des Staatshaushaltes. Seit dem Gemeindekongreß vom Juli 1987 sind verschiedene regionale Gemeindeverbände nach Art deutscher Zweckverbände (gemeinsame Maschinenparks) im Aufbau. Sie werden von der Liga Municipal unterstützt und überwacht. Die weitaus wichtigste Vereinigung stellt die Liga Municipal Dominicana dar. Sie ist in einigen Details zu schildem.

\section{Zur Geschichte der Liga Municipal Dominicana}

a. Die wechselhafte Rechtsentwicklung

Die Liga Municipal wurde im Jahre 1938 - also zu Zeiten der Trujillo-Diktatur - durch Gesetz 49 geschaffen. Bereits dieses Gründungsgesetz läßt die beiden Extreme ihrer Tätigkeit erkennen, zwischen denen der Verband im Laufe seiner Geschichte immer wieder hin und her geschwankt hat:

Auf der einen Seite wird (im Art. 4, so noch in das neueste Organisations- und Verfahrensrecht - Reglamento - 1987 übernommen) in für einen Interessen-Verband klassischer Weise die Förderung der Zusammenarbeit zur Wahrung der gemeinsamen Anliegen und der Verbesserung der durch die Mitglieder (die Munizipien des Landes) zu erbringenden Dienstleistungen zum Verbandszweck erklärt. Dementsprechend ist von irgendwelchen Aufsichtsoder Kontrollrechten gegenüber den Mitgliedern keine Rede - im Gegenteil: Art. 5 legt ausdrücklich fest, daß Beschlüsse der Liga, die die Rechte der Gemeinden betreffen, für diese nur dann verbindlich sind, wenn sie sie in der gehörigen Form als eigene Beschlüsse übernehmen. (Diese Bestimmung ist bis heute nicht förmlich aufgehoben worden).

Auf der anderen Seite fällt auf, daß die Liga von Anfang an nicht als freiwilliger Zusammenschluß der Gemeinden konstituiert war: Sie war durch Gesetz gegründet; die Gemeinden sind Zwangsmitglieder (Art. 1f.); der Innenminister wird mit der Organisation und der Leitung des Gründungskongresses beauftragt (Art. 2). Konsequenterweise ist - übrigens bis 
heute - die Möglichkeit eines Austritts einer Gemeinde überhaupt nicht ins Auge gefaßt worden. Das wäre auch absurd: Seinen Kontrolleur kann man sich nicht aussuchen!

Das immer noch gültige Gemeindegrundgesetz 3455 (in unzähligen, selbst für den juristischen Fachmann kaum mehr überschaubaren Sondergesetzen, oft an versteckter Stelle, verändert) aus dem Jahre 1952 stellt für das offenbar unablässige Bestreben, die Liga zu einem Kontrollinstrument auszubauen, einen ersten Erfolg dar: Uberwachungs- und Kontrollaufgaben, die insbesondere in Finanz- und Haushaltsangelegenheiten genannt werden, kommen in der Regel dem Innenminister, gelegentlich (so etwa Art. 80; 132) auch dem Kongreß (Cámara de Disputados) zu (die Rechnungskontrolle der Gemeinden wird durch die beiden Rechnungshöfe Cámara de Cuentas und Controlaría General wahrgenommen - Art. 38 Ziff.6f.; von einer Rechnungskontrolle durch die Liga ist hier noch keine Rede).

Indessen gibt es Ausnahmen: Von Ausschreibungen zum Kauf beweglicher Güter ist der Innenminister "en su calidad de Presidente de la Liga Minicial Dominicana" zu informieren (Art. 118); die "Entscheidung" (decisión) über die Ausschreibung liegt beim Comité Ejecutivo der Liga (ebenda). Ein gleiches gilt (gemäß Art. 180) für Ausschreibungen an Bauleistungen. Gesetz 4976 (1958) hat hierfür allerdings ein Entscheidungsrecht des Staatspräsidenten eingeführt!) Mit dem Entscheidungsrecht sind konsequenterweise weitreichende Informationsrechte verbunden. Die technischen Aspekte solcher Arbeiten sind durch die Liga in der Form von "Beratungen" zu prüfen; daß solche für die Gemeinden verbindlich seien (so die heutige Lage) wird nicht bestimmt (Buchstabe 1, Art. 180). Das Comité Ejecutivo hat allerdings das weitgehende Recht, die Ausschreibung vollständig an sich zu ziehen "si lo juzga conveniente" (Buchst. k idem).

1954 wird der Liga Municipal durch das Gesetz 3896 der Status einer juristischen Person verliehen. Sie wird vom Präsidenten des Comité Ejecutivo vertreten, von Amts wegen der Innenminister; in einigen Fällen ist ausdrücklich die vorherige Zustimmung des Staatspräsidenten zu Verträgen stipuliert. Schon diese Bestimmungen sind mit den Aufgaben eines Gemeindeverbandes, der Interessen der Mitglieder auch - und gelegentlich: besonders gegenüber nationaler Exekutive und Legislative zu vertreten hat, unvereinbar. Auch die Vorschrift, die Finanzierung der Liga habe aus den Mitteln zu erfolgen, die der Staat als Transferleistungen an die Gemeinden zahle, und deren Höhe werde im Haushaltsgesetz bestimmt, also der Festlegung durch die Liga bzw. ihre Mitglieder entzogen (Art. 3 idem), ist mit einem solchen Selbstverständnis nicht zu vereinen. Ubrigens unterliegt die Liga als Einrichtung bei Käufen ihrerseits der Überwachung der staatlichen Haushaltsverwaltung (Dirección del Presupuesto); bei Kreditaufnahmen ist sogar die Zustimmung des Staatspräsidenten erforderlich. 
Offensichtlich hat die Liga in den fünfziger Jahren ihre Kontrollen und Aufsicht über die Gemeinden enorm ausgeweitet; anders wäre (in Reaktion darauf) Gesetz 5622 vom 14. September 1961 nicht zu verstehen. Es nimmt (Art. 1) dem Staatspräsidenten das Zustimmungsrecht zu einer großen Zahl von Angelegenheiten der gemeindlichen Selbstverwaltung, u.a. zum Haushalt oder zur Aufnahme von Krediten. Dem Innenminister wird (in Art. 2) das Zustimmungsrecht zu gemeindlichen "arbitrios, impuestos o tasas" genommen; auch seine Zustimmungsrechte in Versteigerungsangelegenheiten oder zu solch unzweifelhaften Gemeindeangelegenheiten wie der Namensgebung von Straßen werden aufgehoben. In unserem Zusammenhang ist Art. 3 am wichtigsten: "Die Liga Municipal Dominicana hat kein Recht, in die Angelegenheiten (funcionamiento) der Gemeinden einzugreifen; folglich ist ihre Aufgabe im Hinblick auf diese auf eine rein technische Beratung ... beschränkt". (Die Freigabe des gemeindlichen Abgabenrechts wurde 1966 wieder zurückgenommen.)

1965 bringt eine neue Verschärfung der Nutzung der Liga als Instrument des Staates gegenüber den Gemeinden: Durch Gesetz 603 wird bestimmt, daß der Generalsekretär von der Exekutive, d.h., also dem Staatspräsidenten, ernannt wird. (Offenbar hatte es unerwartete Selbständigkeitsregungen gegeben.) Dem entspricht auch eine wesentliche Verschärfung der Aufsichtsrechte der Liga durch das Gesetz 673 aus dem gleichen Jahr, das das Gesetz 5622 in weiten Teilen zurücknimmt: Die Gemeindehaushaltsentwürfe sind dem Comité Ejecutivo der Liga vorzulegen, das beliebige Veränderungen vomehmen darf, die für die Gemeinden verbindlich sind. (Selbst nachträgliche Verschiebungen von einem Haushaltstitel zu einem anderen sind an die Zustimmung der Liga - Comité Ejecutivo gebunden!) Bedenkt man, daß auch auf Gemeindeebene das Budget ein "Regierungsprogramm in Zahlen" ist, dann ist hierdurch die Gemeindeautonomie im Kem getroffen.

Die offizielle Begründung in der Präambel des Gesetzes, die Gemeindeautonomie werde nicht tangiert, da die Liga nichts anderes sei als die vereinigten Gemeinden selbst, ist weder juristisch noch politisch haltbar:

"Gemeindeautonomie" meint im generellen Verständnis eben das Recht der Einzelgemeinde, im Rahmen ihrer Zuständigkeiten (und selbstverständlich unter Beachtung der geltenden Gesetze) ihrer Angelegenheiten selbst zu regeln. Selbst wenn die Liga im deutschen Sinne ein Gemeindeverband wäre, hätte die Ubertragung wesentlicher Gemeinderechte an sie nichts mehr mit Gemeindeautonomie zu tun. (Inwiefem bliebe etwa die Selbstverwaltung der Stadt Reutlingen unberührt, wenn der baden-württembergische Gemeindetag, in dem natürlich auch Reutlingen vertreten ist, über ihren Haushalt entschiede?). Hinzukommt, daß wegen der personellen und finanziellen Abhängigkeit von der Exekutive die Liga gerade nicht unabhängige Entscheidungen treffen kann (s. dazu unten). 
Neben der Einzelzustimmung zum Haushalt gibt Gesetz 673 der Liga das Recht, Vorschriften zu erlassen "para que los fondos municipales sean invertidos en forma que beneficien a las colectividades...".

Eingeführt wird nun auch (im Art. 4) das Rechnungskontrollrecht der Liga, ohne daß, wie erwähnt, die Uberwachung durch die staatlichen Rechnungshöfe abgeschafft würde. Für die Investitionen der Gemeinden wird die Planung durch die Liga verbindlich, es sei denn, die Gemeinden verfügen über ein technisches Büro mit mindestens fünf Mitarbeitern, von denen mindestens zwei Ingenieure oder Architekten sein müssen. Auch dann kommt der Liga ein Inspektionsrecht der Baustelle zu; diese Inspektionen können in "Empfehlungen" münden. Art. 11 bestimmt, daß Protokolle der Sitzungen der Gemeinderäte innerhalb von fünf Tagen nach der Sitzung an die Liga zu übersenden sind! Ferner ist die Liga jederzeit berechtigt (Art. 12), die Rathäuser zu inspizieren und den Gemeinden und der Regierung Sanktionen zu "empfehlen" sowie zusätzlich die Strafverfolgungsbehörden einzuschalten.

Alle diese Vorschriften sind drei Jahre später (1968) in die Statuten (Reglamento interno) der Liga inkorporiert und sogar noch erweitert worden (z.B. nimmt es - Buchst. i Art. 28 eine Rechtsaufsicht mit Beanstandungsrecht über die Gemeinen in Anspruch). Weitere Einzelheiten mit ähnlicher, teilweise sogar verstärkter Tendenz zur Uberwachung der Gemeinden finden sich im neuesten Statut von 1987.

Schließlich ist noch Gesetz 904 (1978) zu nennen, das bezüglich der Bestimmung des Generalsekretärs den alten Zustand (Wahl durch das Comité Ejecutivo statt Emennung durch die Exekutive) wiederhergestellt hat, mit wenig praktischer Bedeutung.

\section{b. Organe der Liga}

Die Generalversammlung tritt alle vier Jahre zur Wahl des Exekutivkomitees zusammen, das seinerseits, der rechtlichen Regelung nach, den Generalsekretär, den entscheidenden Funktionsträger, wählt. De facto wird dieser jedoch von der Regierung eingesetzt und kann sich ohne das Vertrauen des Staatspräsidenten nicht im Amt halten. Die Organisation mit 265 Mitarbeitern (1987) legt das Schwergewicht auf Inspektion und Kontrolle, nicht auf Planung und Beratung der Gemeinden. (Die Verwaltungsschule wurde bereits diskutiert.) Der Stellenplan weist zahlreiche Ungereimtheiten auf. Der Haushalt, zu vier Fünfteln aus den erwähnten Zuweisungen der Zentralregierung gespeist, ist solide; die Leistungsfähigkeit (also die Effizienz) ist es hingegen nicht. Der Haushalt 1987 betrug ca. 4,8 Mio. RD\$. 


\section{Die Kommunen in der Verfassungs- und Verwaltungswirklichkeit}

\section{Zur politischen Entwicklung der Dominikanischen Republik in den letzten Jahrzehnten}

Die den ausländischen Beobachter am stärksten verblüffende Tatsache der gegenwärtigen politischen Situation in der Dominikanischen Republik ist die Person des Präsidenten, Joaquín Balaguer, als eine der Begleitfiguren der vor Jahrzehnten überwundenen Diktatur Trujillos bekannt. Trotz seines hohen Alters (um 80 Jahre) ist er zweifellos die beherrschende Persönlichkeit der Dominikanischen Republik, in freien Wahlen in sein Amt gekommen (1986) und bisher nicht durch diktatorische Neigungen aufgefallen. Wie ist es zu dieser erstaunlichen Entwicklung gekommen?6

Am 30. Mai 1961 wurde der seit 1930 herrschende Diktator Trujillo erschossen. Hintergrund waren bei einem Teil der Mörder die Begleichung persönlicher oder familiärer Rechnungen mit dem Diktator, bei einem anderen die Tatsache, daß er ihrem eigenen Ehrgeiz im Wege stand. Der Versuch, dem Mord die Aneignung der politischen Macht folgen zu lassen, scheiterte: Der Präsidentensohn Ramfis Trujillo und Joaquín Balaguer, einer der Strohmänner-Präsidenten, durch die Trujillo regiert hatte, machten den Versuch, das Regime ohne den Tyrannen fortzusetzen - erfolglos; die nationale Opposition nahm zu, und auch die Vereinigten Staaten drängten auf Fortschritte hin zur Demokratie.

Schließlich flohen die Mitglieder der Trujillo-Familie (unter Mitnahme von 90 Mio. \$ aus der Staatskasse) aus dem Land, und Balaguer mußte die Macht mit einem siebenköpfigen Staatsrat teilen, der für 1962 Wahlen versprch. Putsch und Gegenputsch zwangen schließlich Balaguer ins Exil, erstaunlicherweise nicht als verhaßten Repräsentanten des abgelösten Regimes, sondern mit beträchtlichem Respekt: Er hatte die vorsichtige demokratische Öfnung ermöglicht, wurde mit der blutigen Unterdrückung Trujillos nicht in Verbindung gebracht und hatte über die Verteilung von Eigentum des Diktators eine eigene politische Gefolgschaft aufgebaut.

Inzwischen waren drei politische Guppierungen entstanden: die Unión Nacional (UCN) als Vertreter der Wirtschaft, die Partido Revolucionário Dominicano (PRD), sozialdemokratisch, und die Bewegung des 14. Juni, castristisch. Juan Bosch, Leiter der PRD, gewann die Wahlen im Dezember 1962. Angetreten mit einem Programm der Wirtschafts- und Sozialreform, löste die Regierung ihr Wahlversprechen nicht ein - konnte es wohl auch nicht. Kirche, Armee, wirtschaftliche Eliten und die amerikanische Botschaft gingen auf Distanz oder zur offenen Opposition über. Das einigende Band war der Antikommunismus: Man

6 Wir stützen uns im folgenden im wesentlichen auf Howard J. Wiarda und Michael J. Kryzanek, The Dominican Republic. A Caribbean Crucible, Boulder (Col.), 1982 und Jan Knippers Black, The Dominican Republic. Politics and Development in an Unsovereign State, Boston u.a., 1986. 
warf Bosch's Regierung Infiltration durch die Kommunisten vor. Im September 1963 putschte die Armee und jagte den Präsidenten ins Exil.

Die zivil-militärische Junta unter Donald Reid Cabral erwies sich als unfähig, das Land zu regieren; im April 1965 putschte der PRD, hatte aber nur einen Teilerfolg: Der Präsidentenpalast wurde besetzt, José Molina Urena, von Verfassungs wegen Interimspräsident, vereidigt - aber die konservativen Militärs starteten einen Gegenangriff. Schließlich griffen die USA militärisch ein, formell zum Schutz amerikanischer Bürger, in Wahrheit zum Anhalten der "Konstitutionalisten", also der pro-Bosch-Kräfte. Die Behauptung der USA, proCastro-Elemente hätten einen kommunistischen Umsturz versucht, war zumindest erheblich übertrieben, wenn nicht falsch.

Im August 1965 kam es schließlich unter amerikanischem Druck zu einem Waffenstillstand der Bürgerkriegsparteien. Interimspräsident wurde Héctor García Godoy, der für den 1. Juni 1966 Neuwahlen ausschrieb. Die psychologischen Folgen der amerikanischen Intervention saßen und sitzen tief: Sind die Dominikaner Herr ihrer eigenen Angelegenheiten? Aber auch die Vertrauenskrise gegenüber der amerikanischen Regierung im In- und Ausland war und ist kaum zu überschätzen.

Die angesprochenen Wahlen - Bosch wagte sich im Wahlkampf kaum aus dem Haus, und Balaguer wurde von den Konservativen der amerikanischen Botschaft und dem Militär unterstützt - endeten mit einem klaren Sieg Balaguers; die sich anschließende Präsidentschaft (bis 1978) war eine der erfolgreichsten der Geschichte des Landes, voran in ökonomischer Hinsicht, wobei freilich massive Schattenseiten nicht übersehen werden sollten (hohe Arbeitslosigkeit und Analphabetenrate, extrem ungleiche Verteilung von Einkommen und Vermögen). Seine Präsidentschaft war vom Stil her - und dies gilt auch für die neue Amtszeit seit 1986 - hoch personalistisch: Sein Damm, seine Agrarreform, seine Wohnungsbauprojekte, seine Schulen, Kliniken, Bewässerungskanäle, Brücken...

Balaguer gewann zwei weitere Wahlen, 1970 und 1974. Die Verschärfung der Wirtschaftskrise (im Gefolge der Olpreiserhöhungen und des Niedergangs der Zuckerpreise), zunehmende Korruptionsvorwürfe und schließlich die Tatsache, daß die Sozialdemokraten in Antonio Guzmán einen auch für die "Oligarchie" und die Amerikaner akzeptablen Kandidaten gefunden hatten, führten schließlich 1978 zum Machtverlust. Balaguer selbst brachte die mit den ersten Resultaten unzufriedenen Militärs dazu, die von ihnen unterbrochenen Stimmauszählungen wieder freizugeben. Guzmán übernahm sein Amt im August 1978.

War die erste Amtszeit Balaguers ein "Trujillismo ohne Trujillo"7? Einige Züge seiner Herrschaft sprechen dafür: Die Herrschaft beruhte wesentlich auf der Unterstützung der

7 So Black, a.a.O., S.42. 
Sicherheitskräfte, die die Bauem ruhig hielt und deren Stimmen "besorgte"; Gewalt gegen politische Gegner (Gefängnis, Exil oder Schlimmeres) spielte bei den Wahlerfolgen eine Rolle; er repräsentierte die mächtigsten Gruppen des Landes; das Auslandskapital wurde mit großzügigen Bedingungen ins Land geholt bzw. im Land gehalten.

Andere sprechen dagegen: Wo bäuerliche Unruhe herrschte (Cibao), wurden Landverteilungsprogramme in die Wege geleitet (von denen freilich wiederum nicht nur Bauem profitierten); der Autoritarismus wurde durch Paternalismus ergänzt; Korruption war zwar verbreitet (und wurde auch vom Präsidenten politisch genutzt), aber Balaguer selbst war im Gegensatz zu Trujillo - an persönlicher Bereicherung uninteressiert; schließlich wäre ein nach Wahlen abtretender Trujillo kaum vorstellbar gewesen.

In unserem Zusammenhang und im Lichte unserer Fragestellung - der Rolle des Präsidenten Balaguer - kann die Präsidentschaft Guzmán und die sich anschließende Jorge Blancos fast als ein Interregnum betrachtet werden. Guzmán war nicht ohne Erfolg, scheint aber in der Person seiner Tochter in massive Korruption verwickelt gewesen zu sein und beging zwei Monate nach der Wahl Blancos (er selbst stellte sich nicht zur Wiederwahl), aber vor der Ubergabe der Amtsgeschäfte, Selbstmord (über die genauen Hintergründe gibt es nur Spekulationen). Jorge Blanco wurde einer massiven Wirtschaftskrise (z.T. durch Exportpreisrückgänge verursacht) nicht Herr; Unruhen wurden mit Gewalt unterdrückt, der IWF verlangte harte Anpassungsmaßnahmen, und das Regime verlor die Wahlen 1986 in einem Sturm des sozialen Protestes und verbreiteter Korruptionsvorwürfe. Sind mit Balaguer unselige Schatten der Vergangenheit auferstanden?

Der Präsident entzieht sich sicherlich einer einfachen Charakterisierung oder Schablonisierung. Natürlich hat er Gewalt, die ihm nutzte, mindestens toleriert (in seiner neuen Präsidentschaft ist davon allerdings weniger die Rede).

Ist von einem solchen Präsidenten die Förderung - oder auch nur Hinnahme - von ihm selbst unabhängiger politischer Kräfte in den Gemeinden zu erwarten? Die Antwort dürfte auf der Hand liegen und wird durch unsere Untersuchung bestätigt: Die zentralistische Tradition der Verfassungs- und Verwaltungsgeschichte wird unter Balaguer nahtlos fortgeführt.

\section{Vom Verhältnis zwischen Staat und Gemeinden}

Unmittelbare staatliche Aufsichtsrechte bestehen im Bereich der Haushaltskontrolle durch die Cámara de Cuentas und die Controlaría General, sodann, ebenfalls im Bereich der Finanzverwaltung, bei Ausschreibungen und Versteigerungen (s.o.). Der deutlich erkennbare Zweck ist, Veruntreuungen von öffentlichen Mitteln, aber auch eine Verschleuderung 
von öffentlichem Vermögen (etwa den Verkauf von Gemeindegrundstücken unter Wert an politische und sonstige Freunde) zu verhindern.

Auf die im einzelnen sehr komplizierten Vorschriften zur Uberwachung gemeindlicher Sonderfonds, die Führung zentraler Register für Verkäufe von Gemeindeeigentum usw. kann hier nicht eingegangen werden.

Über die unmittelbaren staatlichen Aufsichtsrechte hinaus besteht im Jahr 1987 ein politischer Gleichklang von Präsident und Mehrzahl der Gemeinden; eine Einflußmöglichkeit über den Parteiapparat ist zu vermuten.

\section{Zum Verhältnis von Staat und Liga Municipal Dominicana}

Nach Art. 10 Statuten von 1987 ist der Innenminister ex officio Leiter des Organisationskomitees des jährlich einzuberufenden Gemeindekongresses.

Die gleiche Funktion hat er nach Art. 15 für die alle vier Jahre einzuberufende Asamblea General de Municipios.

Wichtiger für die täglichen Geschäfte ist, daß er auch Präsident des Comité Ejecutivo (Art. 17) ist, das üblicherweise alle zwei Wochen tagt.

Da das Comité Ejecutivo aus Politikem besteht, ist - jedenfalls zum gegenwärtigen Zeitraum - der politische Gleichklang mit der Mehrheitspartei gewährleistet. Schlägt der Staatspräsident (natürlich inoffiziell) einen Kandidaten für das Amt des Generalsekretärs vor, dann wird dieser in der Regel gewählt (so beim derzeitigen Amtsinhaber). Die Politisierung des Comité wird durch die Bestimmung unterstrichen, daß die alle vier Jahre zusammentretende Asamblea für vier Jahre das Comité wählt (Art. 16) und dieses (Art. 36) den Generalsekretär bestimmt, dessen Amtszeit mit der politischen Wahlperiode ("Período Constitucional) zusammenfällt.

Weiter sei daran erinnert, daß die Liga mit staatlichen Zuwendungen unterhalten wird. Bei ihren Einzelkrediten, z.B. bei der Vergabe von Kleinkrediten aus dem aus einer amerikanischen Zuwendung stammenden Zirkulationsfonds (Fondo rotativo), respektiert sie die Letztentscheidung des Staatspräsidenten, auch wo dieser - wie hier - ein solches Recht gar nicht hat. 


\section{Zum Verhältnis von Liga Municipal und Gemeinden}

Bezeichnet sich die LMD in der Einleitung ihres Statuts (Art. 2 und 3), in Übernahme der Formulierung ihres Gründungsgesetzes, noch als Vertreter der Interessen und Berater der Gemeinden, so wird dies - zutreffend - in Art. 44, in Rezeption der neueren Rechtsentwicklung, erweitert um die Aufgabe des "Control" und "Fiscalización". (Das alte Statut sprach in seinem Parallelartikel - 35 - nicht davon, obwohl es später als das "Kontrollgesetz" - 673 - erlassen worden war).

Wo die Schwerpunkte liegen, verrät dann unfreiwillig die Beschreibung der Organisationsstruktur (Art. 48), die an erster Stelle die "Unidades de Control y Fiscalización" nennt.

Die entscheidenden Eingriffs-, Kontroll- und Überwachungsrechte der Liga beruhen auf dem oben bereits angesprochenen Gesetz 673 (1965), das fast als "Magna Charta des Untergangs der Gemeindef reiheit" bezeichnet werden kann. Gemeinden unterliegen demzufolge dem unbeschränkten Eingriff in Haushaltsangelegenheiten (Art. 35e); Uberwachung in Personalangelegenheiten (Buchst. i und $\mathrm{j}$ ); der unbeschränkten Verwaltungs- und Rechnungskontrolle (Buchst. k idem); der Kontrolle der Kreditaufnahme sowie der Käufe und Verkäufe von Gütem (Buchst. n idem); Vorschriften der Liga über Kontakte auf Gemeindeebene im In- und Ausland (idem) durch das Comité Ejecutivo; direkten Not-Anweisungsbefugnissen des Generalsekretärs "... para el adecuado funcionamiento... de los Ayuntamientos y Juntas de Distritos Municipales" (Art. 460); sie sind in bestimmten Fällen auf Ankäufe von Gütern durch eine Junta de Compra im Generalsekretariat der Liga (Art. 49) angewiesen. Auch das Recht zu unangekündigten Inspektionen in Ratshäusern besteht weiterhin.

Die Faimeß gebietet es, die Vorschriften zur Förderung der Gemeinden nicht unerwähnt zu lassen. So hat das Comité das Recht und die Pflicht ("Atribución y deber"), Fortbildungsprogramme für Gemeindebedienstete einzurichten (Art. 35h) oder "alle jene Maßnahmen im Bereich von Wirtschaft, Verwaltung und Recht vorzunehmen, die ihm zur Beratung und Hilfe der Gemeinden vom Gesetz übertragen sind" (Buchst. 1 idem; damit kann eigentlich nur das Gründungsgesetz gemeint sein). Auch dem juristischen Laien fällt indes die Präzision der Aussagen über Kontrolle und UUberwachung (von ihrer Zahl abgesehen) und die Unbestimmtheit der Vorschriften über die Förderung der Gemeinden auf. Es unterliegt keinem Zweifel, daß die Liga die Kontrolle wichtiger nimmt als die Förderung, gehindert allerdings gelegentlich durch administrative Mängel. 


\section{Recht und Wirklichkeit}

Der Einwand liegt nahe, in einem stark personenbezogenen politischen System sei die Rechtsordnung von geringem Rang, da die wirklichen Entscheidungsprozesse sich in ganz anderer Form abspielen. Hierzu wäre indes zu bemerken:

a. Zunächst ist zuzugeben, daß weitreichende Kontroll- und Úberwachungsrechte eine Sache, ihre tatsächliche Nutzung mindestens potentiell eine andere ist. Im Laufe ihrer nunmehr fast fünfzigjährigen Geschichte hat der Staat seine Rechte gegenüber der Liga, diese ihre Rechte gegenüber den Gemeinden keineswegs immer in vollem Umfange ausgeschöpft; auch ist die Verschärfung der jeweiligen Kontrollrechte nicht ohne Rückschläge verlaufen, wie deutlich geworden sein dürfte. Insbesondere das Jahr 1961 stellt eine geradezu vehemente Reaktion dar. Vergleicht man indes Ausgangs- und Endpunkt der Entwicklung, dann ist die Gesamttendenz überdeutlich.

Die Ausschöpfung der Durchgriffsrechte des Staates auf die Gemeinden hing im Einzelfall von einer Reihe von Faktoren ab, die wir im Detail nicht nachzeichnen können. Die Persönlichkeiten (bzw. der Herrschafts- und Verwaltungsstil) von Staatspräsident, Innenminister, Generalsekretär der Liga und Bürgermeister der wichtigeren Gemeinden und die allgemeinpolitische Konstellation (Diktatur/Demokratie; Gemeinden mehrheitlich von Regierungspartei bzw. Opposition verwaltet) dürften dabei die wichtigsten sein.

b. Der angeführte Einwand bezieht sich in gewisser Weise auf alle politischen Systeme. In wohl jedem politischen System - und auch im Privatleben - sind "Entscheidungen" und ihre formelle Besiegelung zu trennen. Niemand wird annehmen, die "Entscheidung" über Kauf und Verkauf eines Hauses falle beim Notar - oder der Staatshaushalt werde mit der dritten Lesung des Gesetzes im Parlament "entschieden".

Wichtig ist vielmehr etwas anderes: Entscheidungen fallen stets in einem Rechtsrahmen, der ihren formellen Vollzug strukturiert und äußerste Grenzen des Erlaubten und Möglichen vorgibt. (Das gilt nur dann nicht, wenn die Rechtsordnung insgesamt auf dem Papier steht. Das wird für die Dominikanische Republik niemand behaupten wollen). Hiernach ist es eben ein entscheidender Unterschied, ob Gemeinden unter Beachtung der dafür bestehenden Vorschriften ihren Haushalt selbst beschließen dürfen oder ob eine Úberwachungssituation wie die Liga beliebige Eingriffsrechte hat - selbst wenn sie diese gar nicht nutzen sollte.

c. Die gegenwärtige personelle Konstellation an der Spitze von Staat und Liga gibt keinerlei Hinweis darauf, daß die Überwachungs- und Kontrollrechte etwa nicht extensiv genutzt würden - im Gegenteil. 
d. Die Beratungs- und Hilfsfunktion der Liga gegenüber den Gemeinden ist im Verhältnis zu den Kontrollfunktionen unterentwickelt: Im Einzelfalle ist durch die Spitze der Liga durchaus ein wirksames Eintreten für spezifische Belange von Gemeinden zu verzeichnen, z.B. bei der Steuerbefreiung für gemeindliche Lastwagen. Der gegenwärtige Generalsekretär der Liga ist politisch einflußreich und durchaus zur Nutzung dieses Einflusses im Einzelfalle bereit.

Indessen ist hier keine Systematik oder Routine einer Organisation, sondem ein paternalistisch-wohlwollendes Verhalten einer Einzelperson zu beobachten. Die Beratung durch die Liga ist mangelhaft bis nichtexistent. Am besten dürfte noch die Haushaltsund Finanzberatung funktionieren - wohl deshalb, weil in diesem Bereich ein ausgebauter Kontrollapparat existiert und hier die Ubergange von Kontrolle zu Beratung fließend sind.

Die Liga präsentiert sich also zur Zeit (und als Ergebnis einer langen Entwicklung) als eine Institution, die von der Staatsspitze kontrolliert wird und ihrerseits die Gemeinden kontrolliert. Eine Politik gemeindlicher Dezentralisierung entsprechend dem in der Einleitung angesprochenen weltweiten Trend gibt es in der Dominikanischen Republik zur Zeit nicht.

\section{Zusammenfassung}

Zusammenfassend bleibt festzuhalten: Entscheidungen in der Dominikanischen Republik sind nach Verfassung und Verfassungswirklichkeit hoch zentralisiert. Den Gemeinden wird ein nennenswerter Entscheidungsspielraum nicht zugestanden.

Als wirkungsvolles Kontrollinstrument nutzt der Staat die Liga Municipal. Deren Aufsichtsrechte sind (auch bei Bürgermeistern) inzwischen so internalisiert, daß ein anderer Zustand für viele Verantwortliche im wörtlichen Sinn undenkbar erscheint.

Dementsprechend sind die Gemeindeverwaltungen schwach; sie können viele wichtige Dienstleistungen nur unzureichend erfüllen. Staatliche Stellen springen in die Lücke und verschärfen den Bedeutungsverlust der Gemeinden.

Es sind keine Anzeichen erkennbar, daß ein politischer Wille zu einer Veränderung dieser Situation bestünde - im Gegenteil.

Korrekturen vermag man sich nur im technisch-administrativen Bereich vorzustellen - und hier sind sie auch erwünscht. 
Das skizzierte Grundproblem erscheint den Verantwortlichen gar nicht als solches - es wird nur von ausländischen Beobachtern auf dem Hintergrund ihrer eigenen historischen Erfahrung von Bürgerbeteiligung, Gemeindeselbstverwaltung und Subsidiarität als Problem definiert. 


\section{ABSTRACTS}

In Slow Gallop into the Year of the Horse: New Legal Rules on Birth Planning in the People's Republic of China

By Wolfgang Kessler

Facing a dramatic population growth in the past years symbolized by the passing of the 1.1 billion mark, the People's Republic of China still seeks to curb the increase with stringent birth control legislation. The effectiveness of even such stringent legislation may be doubted due to the strong foundation of social traditions based on economic needs.

Since the national birth legislation has not been enacted yet, the legal rules of the local provinces are still applicable. As one current example, a translation of the Birth Planning Rules of Zhejiang Province of December 1989 is published here.

Local Communities in the Dominican Republic: Constitution and Constitutional Reality in a Centralized Political System

By Jürgen $H$. Wolff

The article examines the current status of the local communities of the Dominican Republic outside of Santo Domingo. Here, like in many countries, communal "autonomy" has been the object of rhetoric. Yet, the political development after the fall of the Trujillo-regime or at least after the end of the civil war and the 1965 US-American intervention could provide a solid basis for more decentralization.

However, apart from improvements in the financial situation of the communities, there is little room for autonomous decisions; well-wishing paternalism is the best the communities can expect from the central government. 